\title{
The Turbulent ISM of Galaxies 10 Gyrs ago: Star Formation, Gas Accretion, and IMF
}

\author{
Loïc Le Tiran and Matthew D. Lehnert \\ Laboratoire d'étude des Galaxies, Etoiles, Physique et Instrumentation \\ GEPI, Observatoire de Paris, UMR8111 du CNRS, 92195 meudon, France
}

\begin{abstract}
The utilization of integral-field spectroscopy has led us to a new understanding of the physical conditions in galaxies within the first few billion years after the Big Bang. In this proceedings, we analyze observations of $\sim 50$ massive galaxies as seen as they were 10 Gyrs ago using SINFONI from the ESO-VLT. We show that the large line width they exhibit can be explained by the intense mechanical energy output from the young stars. We also study the influence of cold gas accretion upon these galaxies: We show that an unrealistic amount of shocked gas would be needed in order to explain the $\mathrm{H} \alpha$ emission from these galaxies through shocks from gas accretion with velocity about the $\mathrm{H} \alpha$ line widths of these galaxies. We also use DEEP2 photometric measurements for a sub-sample of 10 of these galaxies to evaluate their ratio of $\mathrm{H} \alpha$ to FUV flux as a function of their $\mathrm{H} \alpha$ and $R$-band luminosity surface brightnesses. Our data suggests that perhaps their initial mass function (IMF) is flatter than Salpeter at the high mass end, as has been suggested recently for some local galaxies. It may be that high turbulence is responsible for skewing the IMF towards more massive stars as suggested by some theories of star-formation. Much work is however needed to accredit this hypothesis.
\end{abstract}

Keywords. galaxies: high-redshift — galaxies: evolution — galaxies: ISM — galaxies: kinematics and dynamics - infrared: galaxies

\section{Introduction}

Galaxies in the early Universe exhibit many complex features. An interesting example is their large $\mathrm{H} \alpha$ line width, which suggests that a high amount of turbulences is at work in the warm gas of these objects. Many models have been trying to reproduce these different observations, especially with inflows of cold gas (e.g. Dekel et al. (2009)). It is therefore important to distinguish the different contributions to the observables from gas accretion, gravitational instabilities, or mechanical energy output from star formation (which major role is emphasized in Lehnert et al. (2009)). The IMF is another important underlying parameter which determines some of the most obvious characteristics of galaxies as observed in the local universe. Many recent studies have been studied this assumption, using for example the quantity $F_{\mathrm{H} \alpha} / f_{\mathrm{FUV}}$ (but only in the local universe, e.g. Meurer et al. (2009)). Perhaps though, galaxies in the early Universe, with their generally more extreme properties are interesting targets for studying star formation under extreme physical conditions, high turbulence and thermal pressures, which might impact the overall shape of the IMF.

In this proceedings we use SINFONI observations on the ESO-VLT of a sample of fifty galaxies around $\mathrm{z} \sim 2 \pm 1$. This sample will be described in details in Le Tiran et al. (2011, in preparation). 


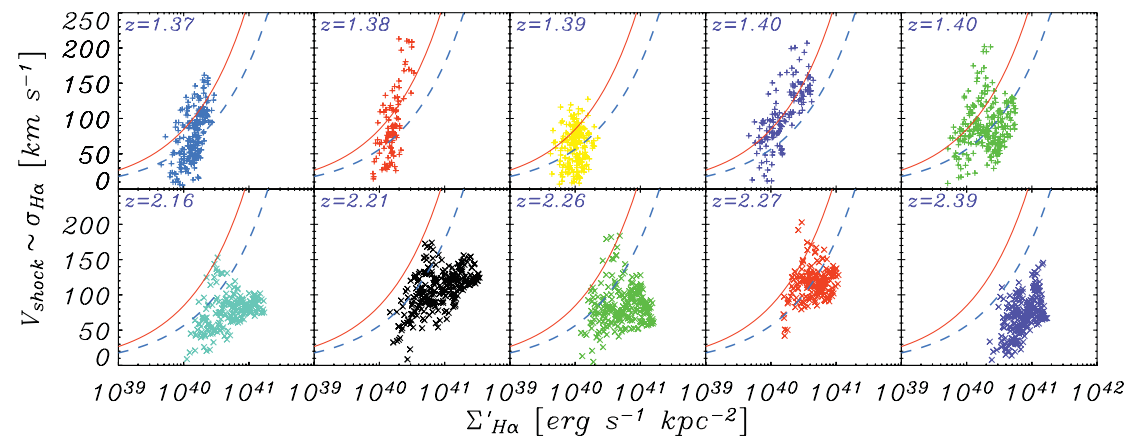

Figure 1. Observed $\mathrm{H} \alpha$ surface brightness values (in $\mathrm{erg} \mathrm{s}^{-1} \mathrm{kpc}^{-2}$ ) as a function of $\mathrm{H} \alpha$ line width (in $\mathrm{km} \mathrm{s}^{-1}$ ) for a representative sub-sample of 10 galaxies. Redshifts of these galaxies are labeled in the upper-left corner of each panel. The solid lines show two simple relationships of the form $\sigma=\sqrt{ } \epsilon \dot{E}$, where $\epsilon$ is the efficiency of coupling between the energy injected and the ISM: $\sigma=200 \sqrt{ } \Sigma_{S F R} \mathrm{~km} \mathrm{~s}^{-1}$ (in dashed blue), $\sigma=300 \sqrt{ } \Sigma_{S F R} \mathrm{~km} \mathrm{~s}^{-1}$ (in plain red).

\section{What is driving the internal motions of these galaxies?}

Galaxies at high redshift exhibit surprisingly high values of their $\mathrm{H} \alpha$ line widths. During the last years several explanations have been suggested for the underlying physical mechanism that drives these high dispersions. They could be for example generated by Jeans instabilities in the gas, by the general gravitational instabilities in the cooling gaseous disks of galaxies, or by the infall of gas from cosmic accretion (an effect studied in more details in Le Tiran et al. (2011, submitted) and briefly in Section 3). However, as developed in Lehnert et al. (2009), none of these proposed mechanisms appears sufficient to reproduce the high velocity dispersions we observe.

Lehnert et al. (2009) suggested that the dynamics of the emission line gas inside the galaxy is powered by the mechanical energy of star formation. If we make the hypothesis that the mechanical energy output of the star formation is roughly conserved in the ISM, by using a simple dimensional analysis we expect the velocity dispersion to be proportional to the square root of the energy injection rate of the stars, $\mathrm{dE}_{S F} / \mathrm{dt}$. This relationship is over-plotted on Fig. 1: using a simple scaling law based on the starformation rate per unit area and the velocity dispersion in the warm neutral/ionized gas in the disk of the MW and other nearby galaxies. This simple toy-model, which has the advantage of using no free parameter, reproduces the observed trend between the velocity dispersion and the star formation intensity (a more detailed analysis can be found in Lehnert et al. (2009)). It is therefore the star formation itself that is controlling the dynamics of the emission line gas.

\section{Can shocks induced by cosmological gas accretion produce the observed $\mathrm{H} \alpha$ brightnesses?}

Gas accretion onto galaxies is undoubtedly a complex process. To explain cold gas accretion, the gas has to lose its energy and momentum through radiation and shocks upon hitting the ISM of the galaxy. Can we see direct evidence for this in our integral field $\mathrm{H} \alpha$ data sets?

If the infalling gas dissipates energy by colliding with gas already in a galaxy disk, we can estimate the rate at which it dissipates using various complementary shock models (Raymond (1979), Shull \& McKee (1979), Allen et al. (2008)). We will assume that the 


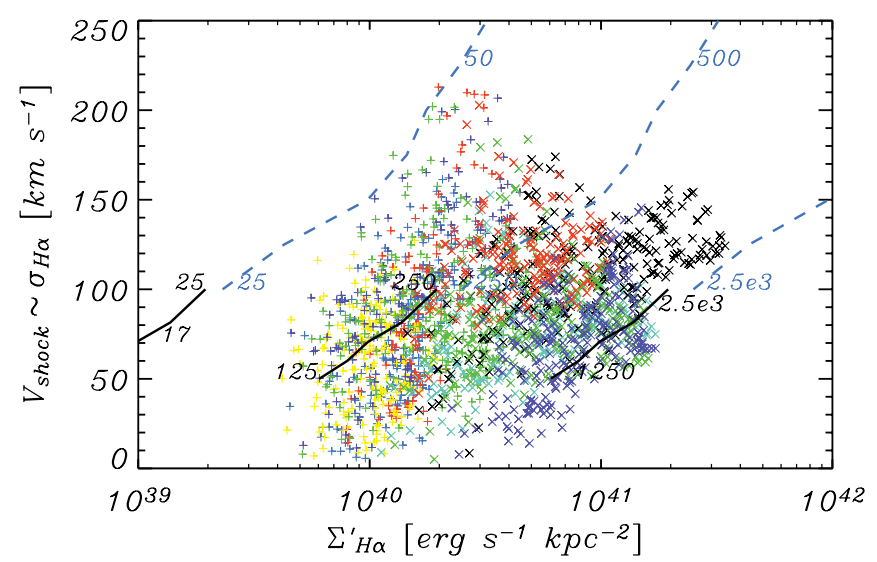

Figure 2. Observed $\mathrm{H} \alpha$ surface brightness values (in $\mathrm{erg} \mathrm{s}^{-1} \mathrm{kpc}^{-2}$ ) as a function of $\mathrm{H} \alpha$ line width (in $\mathrm{km} \mathrm{s}^{-1}$ ) for a representative sub-sample of 10 galaxies. Colors and symbols are the same than in Fig. 1. Lines correspond to the $\mathrm{H} \alpha$ surface brightnesses which would be generated by shocks with a velocity equal to the velocity dispersion of the $\mathrm{H} \alpha$ line. Models from Raymond (1979) are in plain black and from Allen et al. (2008) in dashed blue. The numbers along each line indicate the surface accretion rate (in $\mathrm{M}_{\odot} \mathrm{yr}^{-1} \mathrm{kpc}^{-2}$ ) necessary to produce the observed $\mathrm{H} \alpha$ surface brightnesses. The 3 sets of lines are for a different pre-shock density, 10, 100, $1000 \mathrm{~cm}^{-3}$, from left to right.

observed velocity dispersion in distant galaxies is about the shock velocity range $(\sim 50$ $\left.250 \mathrm{~km} \mathrm{~s}^{-1}\right)$. The underlying assumption is that all the accretion energy is converted into supersonic turbulence which then dissipates in fast shocks. We can then estimate the amount of mass that would be need to be shocked to explain the observed surface brightnesses as a function of dispersion. The results are shown in Fig. 2. We find that to explain the surface brightnesses with these shock models, the necessary mass flow rates through the shocks are unrealistically high, implying accretion rate densities of over a few hundred $\mathrm{M}_{\odot} \mathrm{yr}^{-1} \mathrm{kpc}^{-2}$. Therefore, over a dynamical time of about 200-300 Myrs, the amount of gas that would need to be shock heated is of the order of $10^{13} \mathrm{M}_{\odot}$, two orders of magnitude larger than the total ISM mass. A more complete analysis of the influence of the gas accretion, and its importance relative to $\mathrm{H} \alpha$ emission from star formation, will be presented in Le Tiran et al. (2011, submitted).

\section{Evidence for a top-heavy IMF?}

This section focuses on a sub-sample of 10 galaxies, for which we have DEEP2 data. We can therefore estimate $F_{\mathrm{H} \alpha} / f_{\mathrm{FUV}}$, a quantity which is sensitive to the relative fractions of high mass (ionizing) to lower mass (non-ionizing) stars. The $\mathrm{H} \alpha$ emission traces the rate of ionization which is dominated by the massive $\mathrm{O}$ stars, over $20 \mathrm{M}_{\odot}$, while the FUV emission mainly traces somewhat less massive $(\mathrm{O}$ and $\mathrm{B})$ stars, over $3 \mathrm{M}_{\odot}$. Making these measurements for our sample, we find that these distant galaxies "extend" the relationship found in Meurer et al. (2009) to higher surface brightnesses and more extreme ratios, as seen in Fig. 3. This suggests that the relationship between $F_{\mathrm{H} \alpha} / f_{\mathrm{FUV}}$ and surface brightness is one of the fundamental scaling relationships for galaxies as emphasized by Meurer et al. (2009). The high values we obtain for $F_{\mathrm{H} \alpha} / f_{\mathrm{FUV}}$ and high surface brightnesses may suggest a top-heavy IMF in these galaxies. Due to the lack of the necessary data, the relationship we present is unfortunately unextincted. Our 

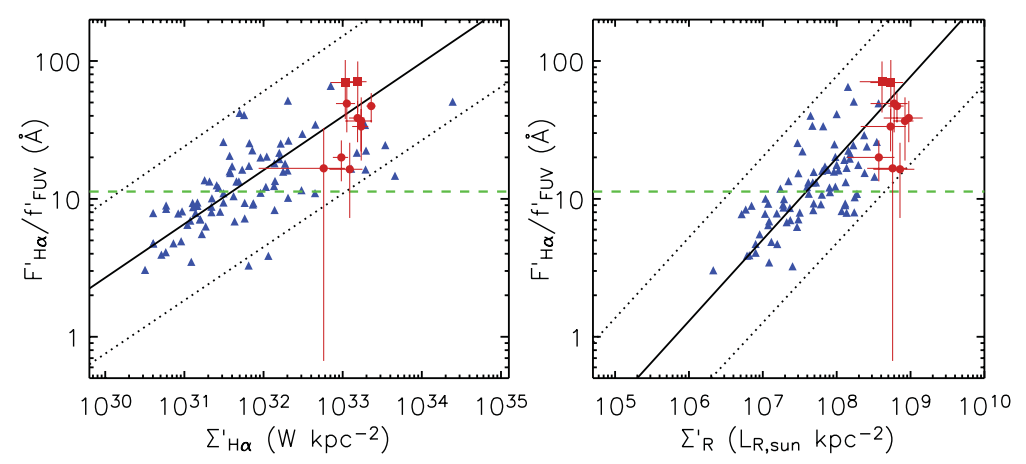

Figure 3. The ratio of $\mathrm{H} \alpha$ line flux to FUV flux-density as a function of the $\mathrm{H} \alpha$ effective surface brightness (right panel) and as a function of the $R$-band effective surface brightness (left panel). Blue triangles are the local galaxies data from Meurer et al. (2009), red circles are data from this work as well as red squares that represent AGN. The uncertainties are $3 \sigma$. Galactic and internal dust absorption have not been taken into account in any of these data. The solid line shows the iteratively clipped least squares fit to the Meurer et al. (2009) data, while the dotted lines show the final clipping limits. The green dashed line corresponds to the $F_{\mathrm{H} \alpha} / f_{\mathrm{F}} \mathrm{VV}$ fiducial value of $F_{\mathrm{H} \alpha} / f_{\mathrm{FUV}}$ for a constant star-formation rate.

modeling shows that neither bursts of star-formation nor changes in the metallicity can explain our measurements.

To study the impact of varying high-end of the IMF, we used Starburst99 (Leitherer et al. (1999)) to build synthesis models for IMFs with varying slopes from $\gamma=1.25$ to 3 . and varying upper-masses from $M=60 \mathrm{M}_{\odot}$ to $120 \mathrm{M}_{\odot}$. While variations of the upper-mass limit of the IMF cannot explain our measurements, a flatter slope would be a possible explanation to the high values of $F_{\mathrm{H} \alpha} / f_{\mathrm{FUV}}$. These results, although preliminary, suggest that perhaps the very particular physical conditions at work in high redshift galaxies favor a top-heavy IMF. Additonal data is needed to investigate this hypothesis.

\section{Conclusion}

Galaxies within the first few Gyrs in the age of the universe provide extreme conditions which may impact the nature of galaxies and their star-formation. Galaxies in our sample have star formation rate more than one order higher than typical galaxies in the local Universe, creating the high velocity dispersions we observe in ionized gas. In the case of cold flows of gas accreting upon a galaxy, the amount of shocked gas that would be needed to explain such $\mathrm{H} \alpha$ emission would be unrealisticaly high. Using the relationship between $F_{\mathrm{H} \alpha} / f_{\mathrm{FUV}}$ and surface brightness as a diagnostic, we find that perhaps there is a top-heavy IMF in these high redshift galaxies. This may be a result of the relative high pressures and turbulence we find for galaxies at high redshift.

\section{References}

Allen, M. G., Groves, B. A., Dopita, M. A., \& Sutherland, R. S., et al. 2008, ApJS, 178, 20

Dekel, A., et al. 2009, Nature, 457, 451

Lehnert, M. D., Nesvadba, N. P. H., \& Le Tiran, L., et al. 2009, ApJ, 699, 1660

Leitherer, C., et al. 1999, ApJ, 123, 3

Meurer, G. R., et al. 2009, ApJ, 695, 765

Raymond, J. C. 1979, ApJS, 39, 1

Shull, J. M. \& McKee, C. F. 1979, ApJ, 227, 131 